\title{
Reflexiones en torno al trabajo actual y sus repercusiones en la (de) construcción de la sociedad contemporánea
}

BEATRIZ TORRES GÓNGORA ${ }^{56}$

Resumen: El artículo estudia el trabajo en las sociedades contemporáneas a partir de los cambios suscitados en los últimos treinta años, como parte de las intensas transformaciones económicas, políticas, sociales y culturales derivadas del reajuste capitalista a escala global y la crisis en la que se encuentra el sistema. El texto desarrolla reflexiones sobre el papel del trabajo en la sociedad actual, el lugar que éste ocupa en la vida de las personas y cuál sería su importancia en la cotidianeidad. El artículo analiza algunos indicadores centrales del mundo del trabajo en seis países de América Latina: Argentina, Brasil, Chile, Colombia, México y Perú. Filnalmente, el estudio relaciona la problemática del trabajo con los objetivos y prácticas de la política social latinoamericana.

Palabras-Clave: trabajo; política social; América Latina

\begin{abstract}
This article is about work in contemporary societies from the changes happened in the last thirty years as part of the intense economic, political, social and cultural transformations derived from the capitalist readjustment in global level and the crisis in which this system is presently. The text develops reflections on the role of work in today's society, the place it occupies in the life of people and its importance in daily life. The article analyzes some central indicators of the world of work in six Latin American countries: Argentina, Brazil, Chile, Colombia, Mexico and Peru. Finally, the text relates the problematic of work with the goals and practices of Latin American social policy.
\end{abstract}

Keywords: work; social policy; Latin America.

\section{Introducción}

El tema que nos ocupa es el trabajo en las sociedades contemporáneas, tópico de gran importancia ante los cambios suscitados en los últimos treinta años, como parte de las intensas transformaciones económicas, políticas, sociales y culturales derivadas del reajuste capitalista a escala global y la crisis en la que se encuentra. La interrogante que guía el texto se dirige a reflexionar si ¿puede reconocerse al trabajo [asalariado] como el centro de la cuestión social tal como señaló Castel, (1997) a mediados de los noventa o como él mismo replanteó, en 2009, la cuestión social se trasladó a lo urbano ${ }^{57}$ y a lo étnico ${ }^{58}$ ? O tendrá razón

56 Profesora- investigadora de la Unidad de Ciencias Sociales del Centro de Investigaciones Regionales "Dr. Hideyo Noguchi" de la Universidad Autónoma de Yucatán, México. E-mail: beatriztg4@hotmail.com y tgongora@uady.mx

57 El mismo Castel, 2010, recupera los planteamientos de Jacques Donzelot en el sentido de la transferencia del trabajo hacia el territorio como el centro de la cuestión social porque, en especial, es en el espacio urbano donde se ubican "las líneas de escisión, las oposiciones y los conflictos que estructuran la vida social, y donde se cristalizan las principales desigualdades" (Castel: 42). Bajo este planteamiento el trabajador es sustituido por el habitante como principal interlocutor de los poderes públicos.

58Explicada por el mismo autor (Castel, 2010), para el caso de Francia, como resultado de grandes olas migratorias cuyos individuos sufren un doble descrédito al mismo tiempo: el proveniente de su etnia y el de su descalificación social por el mismo motivo. 
Bauman (2000) cuando afirma que el consumo ha logrado desplazar al trabajo y ocupar su lugar ${ }^{59}$ como elemento vertebral de la vida social?

Cuando hablamos de la cuestión social, espero estemos de acuerdo en entenderla como el motivo central alrededor del cual se mueve la sociedad, con tal poder que en lo negativo puede amenazar su cohesión. O sea, es el meollo de la preocupación social en determinado momento. En el caso del trabajo, el motivo que lo eleva a la palestra es su capacidad para inscribir, insertar, vulnerar, excluir o, en palabras del mismo Castel, desafiliar a los individuos del resto de la sociedad.

La primera parte del texto estará dedicada a exponer algunas reflexiones acerca del papel del trabajo en la sociedad actual, el lugar que ocupa en la vida de las personas y en cómo expresa su importancia en la cotidianidad; asimismo se ubican las principales transformaciones del mundo del trabajo, en las últimas décadas y los grupos y regiones más afectadas. En el apartado dos, de forma somera, se presenta el comportamiento de algunos indicadores centrales, correspondientes al mundo del trabajo en seis países de América Latina: Argentina, Brasil, Chile, Colombia, México y Perú. En realidad, se trata de una rápida mirada a las estadísticas cuyas limitaciones son innegables ${ }^{60}$. Lo cualitativo, la investigación de terreno, está acotado a la revisión de selecta bibliografía especializada de los países examinados. Finalmente, en un tercer momento, a manera de conclusión, buscaré relacionar tal problemática del trabajo con los objetivos y prácticas de la política social latinoamericana.

\section{I - El desafío del trabajo en el centro de la problemática social}

A nivel individual contar con un trabajo que permita afrontar los costos de los bienes y servicios necesarios para la subsistencia del día a día, se convierte en un reto mayor para los 17 millones de latinoamericanos desempleados, cifra estimada para 2012 ${ }^{61}$ por la Comisión Económica para América Latina y el Caribe (CEPAL). Desempleados que constituyen alrededor del 7\% de la PEA de la región. Adicionalmente, se sabe también de varios millones más de personas en situación de subempleo por razones de mercado. Esto es, son individuos que se desempeñan laboralmente menos horas debido a una demanda insuficiente del factor trabajo. Ellos suelen obtener magros ingresos y frecuentemente forman parte del creciente contingente de los pobres ${ }^{62}$. Grave también, son las altas tasas del desempleo juvenil ${ }^{63}$, sector de la población que junto con las mujeres ${ }^{64}$ y los grupos étnicos ${ }^{65}$ constituyen los segmentos de la población económicamente activa (PEA) con mayores dificultades para acceder a un puesto de trabajo.

Para la mayoría de las personas, tener y ejercer una ocupación es el medio por excelencia de obtener recursos monetarios y no monetarios para sustentar su vida diaria pero también es fuente de satisfacción o sufrimiento personal. Adicionalmente, trabajar se ha convertido en condición para la inserción y reconocimiento social de los individuos, motivo de aceptación o rechazo como ciudadano vigente, medio para ocupar un lugar en la sociedad

\footnotetext{
59Al respecto, ver Bauman, 2002 que al seguirle la pista a la ética del trabajo plantea el paso de una sociedad de productores a otra de consumidores donde la estética del consumo toma el lugar de la ética del trabajo.

60 La información proviene de diferentes encuestas de fuerza laboral levantadas en cada uno de los países y sistematizadas por la Organización Internacional del Trabajo (OIT).

61 Según pronóstico de la CEPAL, "Habrá 17 millones de desempleados en América Latina en 2012".

62Según cálculos de la CEPAL, el número de pobres latinoamericanos pasó de 180 millones en 2008 a 189 millones en 2009 principalmente a causa de la crisis económica global. En “Cepal: 800000 más caen en pobreza extrema en México y Centroamérica. El economista, 21 de enero de 2010.

63De acuerdo a las cifras de la OIT para 2011, la desocupación juvenil fue tres veces más que la registrada en los adultos (14.9\% para los primeros y $5.0 \%$ para los segundos).

64Según la OIT (2011) el desempleo femenino equivale a 1.4 veces que el masculino.

65Frecuentemente discriminadas, excluidas del trabajo o esclavizados.
} 
y ser visibles como miembros de ella. En algunos países también es fuente de derechos sociales dada la vinculación que se hace del trabajo asalariado con la protección social.

De aquí la tragedia que puede ser para los individuos el hecho de no contar con una ocupación. Tragedia con hondas repercusiones en la persona que lo vive, principalmente por los efectos psicosociales que le acarrea. Pero la falta de ocupación que padece también afecta a la familia de la que forma parte, al convertirse en un ser dependiente y, en muchos casos, obliga a buscar la incorporación temprana o tardía de otros miembros de la familia; igualmente afecta a la comunidad en la que se encuentra inscrito; a la organización sindical que, en caso de existir, se ve minada; al país en cuestión y a la sociedad en su conjunto. ¿por qué es tan grave la escasez de ocupación?

De vez en vez, aunque con mayor frecuencia, la prensa contemporánea detalla lo que ya se sabe por estudios especializados: el incremento de la depresión y el suicidio de personas desempleadas. Tal vez es el hecho de que la noticia, en el caso de los suicidios, ponga nombre, edad, nacionalidad, medio utilizado para hacerlo y demás pormenores ${ }^{66}$ cumple el cometido de llamar la atención hacia esta problemática tan cotidiana pero, al mismo tiempo, tan callada por el cuestionamiento social que puede generar sobre quien la padece pues siempre es posible atribuir la responsabilidad al mismo individuo bajo argumentos de pereza, falta de pericia, de preparación, de voluntad, etc. Se trata de esa distinción social negativa que tanto lastima y vulnera a las personas.

Por el contrario, en la dimensión social, el silencio se rompe, dada la recurrencia y gravedad del déficit de empleo que ha alcanzado tintes escandalosos, convirtiéndose en motivo de descontento, protesta y conflicto social como atestiguan variados y ascendentes movimientos, en varias partes del orbe ${ }^{67}$.

Si bien en el centro de los conflictos sociales se encuentra el cuestionamiento hacia el sistema económico capitalista, sobresale el problema de la falta de empleo de calidad -lo que existe es ocupación precaria- como su consecuencia más grave, cuyo origen se ubica en la preeminencia del mercado sobre lo social, mismo que se expresa en los actuales criterios de productividad, caracterizados por depredar tanto los recursos naturales como al hombre mismo, quien es tratado como un bien abundante, fácilmente reemplazable y desechable; en el predominio del capital financiero sobre el productivo, en el uso inhumano de las nuevas tecnologías en el ámbito de la producción de bienes y servicios ${ }^{68}$ y la encarnizada competencia por los mercados a nivel global, entre lo más importante.

En el caso de América Latina, adicionalmente se suman las consecuencias de un largo colonialismo, todavía palpable en la vida diaria de sus poblaciones, como una forma más de sometimiento (Quijano, s/f; De Sousa, 2010) que también es trasladada a la esfera laboral.

Si bien, la falta de empleo tiene una dimensión individual, o sea, es Panchito, Irene o Asunción los que están en paro, tales situaciones evidencian problemas estructurales del modo de acumulación capitalista que mantiene en reserva a una gran masa de población desempleada, lo que junto al constante incremento de la productividad, favorecida por el amplio desarrollo tecnológico, tiende a reducir la demanda de fuerza de trabajo, de trabajo vivo. De esta manera, la falta de empleo se erige como elemento constitutivo de su dinámica,

66"Se incrementa suicidios en Italia por crisis económica". El Universal, Sección El Mundo, 20 de agosto de 2012; "Suicidio de jóvenes por falta de empleo" por Melvin Áreas Trinchera de la noticia. 20 agosto de 2012; "Se suicida por falta de empleo" Noticaribe Cozumel, 17 de junio de 2010; "Se suicida piloto de Aeroméxico por falta de empleo" Noticias MVS, 18 de octubre de 2011.

67Véase, Harvey y otros, 2012.

68De acuerdo a Girardi (2004:60) el desarrollo tecnológico se refiere a la aceleración de los medios de transporte y perfeccionamiento vertiginoso de los medios de comunicación, particularmente de Internet; progresos que suprimen las distancias espaciales y temporales y transforman al mundo en una aldea global. 
que a su vez configura el contexto en el que se ofrecen o se cancelan las oportunidades de ocupación de los individuos que la integran.

Todas las situaciones individuales se encuentran enmarcadas en un contexto social que constriñe el actuar individual dando, de esta manera, una fisonomía al mundo del trabajo en un espacio y tiempo determinado, donde lo individual alimenta las tendencias observadas para el conjunto de la sociedad, en este caso, del ámbito laboral. Pero, al mismo tiempo, tal conjunto delimita los bordes de posibilidades del actuar individual. Se trata de una dinámica compleja, interactiva, heterogénea, a veces, contradictoria, entre lo macro, meso y micro, entre el individuo, la familia y la sociedad, que involucra dimensiones económicas, políticas, sociales y culturales; donde la racionalidad económica de los actores únicamente alimenta una parte de sus decisiones. Las otras provienen de los demás ámbitos concurrentes. En este sentido, "lo laboral" se construye con hechos económicos, políticos y sociales pero también desde la cultura y la subjetividad.

En el actual contexto de escasez de empleo, pero sobre todo, de empleo de calidad, una de las problemáticas que destaca es la propagación del miedo a la exclusión laboral ${ }^{69}$. Entre los trabajadores cunde el miedo a los despidos derivado de la experiencia de formar parte de un colectivo que lo padece, mientras que los que aún están en búsqueda de una ocupación suelen ofrecer, por el mismo motivo, una amplia disposición a aceptar casi cualquier condición a cambio de un puesto de trabajo. En este sentido, el miedo a estar desocupado se convierte en mecanismo eficaz de disciplinamiento y precarización de las condiciones de uso y retribución de la mano de obra, tanto para los que están trabajando como para los que no lo están, provocando inseguridad, frustración, descontento, rabia, desaliento, desesperanza, entre otras emociones, así como efectos de deterioro de la salud de quienes lo padecen, principalmente por el estrés constante al que se encuentran sometidos.

Visto desde la dimensión de la sociedad, la falta de empleo amenaza su cohesión constituyendo el centro de los problemas económicos, políticos y sociales, tal como apuntara la OIT desde 200570 en la IV Cumbre de las Américas que bajo el lema "Crear trabajo para enfrentar la pobreza y fortalecer la gobernabilidad democrática" instó a la reflexión y a la revisión del papel del trabajo en la sociedad globalizada, como tarea indispensable para conjurar el conflicto social. Igualmente la campaña de procuración del trabajo decente ${ }^{71}$, emprendida por el mismo organismo desde 1998, surge con la intención de proveer un sentido ético y humano al trabajo, aportando en la misma vía.

Con tales planteamientos se cuestiona la inevitabilidad de la explotación del hombre por el hombre, de la predominancia del mercado sobre la sociedad y su degradación como signo inevitable de la globalización en marcha. Pero el hecho contundente que ha cuestionado la dinámica actual del sistema económico y sus consecuencias, entre las que destaca la falta de oportunidades de trabajo de calidad, es el malestar popular ${ }^{72}$, expresado en las luchas sociales suscitadas durante la década de los noventa y los años siguientes, sobresaliendo el 2011 por su alta conflictividad social ${ }^{73}$.

69Vale hacer notar que Weller (2011) distingue cuatro formas de exclusión, mismas que el autor presenta como obstáculos para acceder a un empleo de calidad: del mercado laboral, del empleo, del empleo productivo y del empleo de buena calidad en sectores de alta y mediana productividad. Cada una de ellas afecta diferencialmente a los diversos segmentos de la población económicamente activa y representa retos específicos para grupo. La exclusión al que nos referimos equivaldría a la que este autor denomina exclusión del empleo y puede evaluarse mediante la tasa de desempleo.

70 OIT, 2006.

71Según la OIT, el trabajo decente es un trabajo productivo, justamente remunerado, ejercido en condiciones de libertad, equidad, seguridad, capaz de garantizar una vida digna" sustentado en cuatro ejes: respeto a los derechos del trabajo, promoción del empleo de calidad, extensión de la protección social y diálogo social.

72 Un elemento inesperado en tales movimientos es la participación de sujetos alejados de los sindicatos y partidos políticos quienes tradicionalmente habían encabezado los movimientos sociales, se trata de amas de casa, los jóvenes, personas de la tercera edad y población en general.

73Al respecto, véase Varios autores. Occupy, 2012. 
Adicionalmente, la crisis iniciada como crisis financiera, a finales de 2008, en los Estados Unidos de América, produjo una grave desaceleración económica en ese país, misma que se propagó aceleradamente, cual ondas concéntricas, por el resto del orbe y, contra los pronósticos de organismos internacionales, sus efectos continúan manteniendo la contracción económica, la reducción de las inversiones productivas, la disminución del gasto social y el deterioro del mundo del trabajo ya de por sí muy afectado por el proceso de globalización que trajo consigo transformaciones a diversos niveles, entre los que destaca:

a) una nueva manera de producir, sustentada en la fragmentación de los procesos productivos a escala global y el traslado de segmentos de los mismos a zonas de menores costes, generando procesos de trasnacionalización, desterritorialización y reterritorialización del capital, y en menor medida, del trabajo. Tales procesos, han sido posibilitados por el desarrollo de las tecnologías de información y comunicación que han dado un nuevo sentido al tiempo y a la distancia.

b) el ámbito de los procesos de trabajo también ha sufrido importantes transformaciones asociadas con la búsqueda de restitución de la tasa de ganancia disminuida, en parte, por el agotamiento del modelo taylorista fordista que encontró sus límites en nuevos requerimientos del mercado orientados a la variedad y calidad de los productos.

La estandarización de la producción y de las tareas, sostenidas en una estricta división del trabajo y, posteriormente, en la cadena de montaje que fijó al trabajador a su puesto, determinándole el ritmo de producción, y con ello, la intensidad del trabajo, dio como resultado una alta especialización de la mano de obra, especialización que funcionó como base de la productividad de la llamada producción en masa.

Sin embargo, la rigidez que caracteriza a este sistema productivo se volvió inviable ante la demanda diferenciada, mientras que sus límites sociales emergieron, en forma de desafección al trabajo, expresada en ausentismos, rotación de personal, aumento de desperfectos y desperdicios, presiones sindicales, conflictos laborales, etc. De parte del trabajo, su implicación se resquebrajó por el fuerte desgaste al que se encontraba sometido, principalmente en el trabajo industrial de los llamados países desarrollados y sus filiales ubicadas en los países de menores costos salariales.

La naturaleza resultante de esta forma de trabajar, caracterizada por la prescripción detallada de las tareas, la repetición de las mismas, su control mediante los estudios de tiempos y movimientos, la vigilancia directa y el uso del cronómetro como auxiliar para ello convirtió al trabajo en rutina y permitió la incorporación de trabajadores descalificados al hacerse innecesario sus saberes, producto de largos años de oficio. Esta es una de las consecuencias ampliamente documentadas de esta forma de trabajar (Braverman, 1974; Coriat, 1979) 74 .

La emergencia del sistema de producción ligera o ajustada, surgida en la planta japonesa de la automotriz Toyota fue el evento que destrabó tal nudo, cuando menos en lo que se refiere a los países industrializados, cuyas empresas encaminaron sus esfuerzos para beneficiarse de su potencialidad para responder, de forma satisfactoria, a las nuevas exigencias del mercado en cuanto a variedad y calidad de los productos. Adicionalmente, sus promotores ofrecían disminuir o eliminar desperdicios, tan característico de la forma de producción en masa.

Como bien señaló Coriat (1992), se trataba de "pensar al revés" los principios productivos del taylorismo fordismo y flexibilizar el proceso productivo y el uso de la mano

74Aunque una gran parte de la estructura productiva de los países latinoamericanos desconocía este sistema productivo y continúo produciendo bajo formas pretayloristas, caracterizadas por poca planeación y mucha improvisación. 
de obra. La demanda de polivalencia de la fuerza de trabajo compactó tareas, puestos, departamentos, al mismo tiempo que buscó la implicación de los trabajadores mediante diversos mecanismos como los bonos e incentivos económicos, algunos otorgados de forma grupal. De esta manera, la retribución de la fuerza de trabajo también se volvió incierta y dependiente de su desempeño. Así la flexibilización del trabajo en su contratación, uso y retribución se fue extendiendo y profundizando como uno de los mecanismos principales, utilizados por el capital para abaratar costos de producción y abonar a la productividad en el nuevo contexto de mercados inciertos y competitivos.

Uno de los cambios más importantes, observados entre un sistema a otro, es la forma de concebir y, por ende, de tratar al elemento humano en los procesos de trabajo. Mientras que en el taylorismo fordismo se buscó eliminar la inteligencia de los procesos productivos e instaurar el hábito como la base de las tareas, en el toyotismo se gestiona el uso acotado de su iniciativa y la puesta en práctica tanto de sus capacidades físicas y mentales como de las emocionales, principalmente cuando se trata de la procuración de servicios.

Vale aclarar que tanto los principios productivos del taylorismo-fordismo como del toyotismo adquieren especificidades del país y el momento de su implementación; como cualquier modelo no puede ser transferido tal cual de una región a otra, ni existen en el vacío.

c) El desplazamiento de la producción industrial hacia la de servicios y bienes inmateriales es otra de las características en esta etapa del capitalismo; junto con ello, el conocimiento y la información saltan a la arena del mundo productivo como elementos estratégicos para su desarrollo. A nivel de las grandes firmas, puede observarse la extensión del trabajo sustentado en capacidades cognitivas que transfieren mayor valor agregado a los productos y servicios en medio de una acelerada y disputada carrera por la innovación de productos y procesos.

d) La incorporación de los adelantos tecnológicos -informática, robótica, biotecnología- a los procesos productivos dio nuevo impulso a la productividad, al mismo tiempo que desplazó trabajadores. Así, el uso de la tecnología informática se ha incorporado a infinidad de procesos productivos como una herramienta central que posibilita a los trabajadores la realización de varias tareas a la vez, así como su control y vigilancia por medios electrónicos, sin importar la distancia que puede ser transoceánica o trascontinental. Asimismo, la extensión de la tecnología en el sector servicios ha impactado negativamente al empleo al automatizar gran parte de las tareas, eliminando puestos de trabajo, mediante la modalidad del autoservicio.

De esta manera, también se ha requerido la participación activa de los consumidores en la distribución y consumo, tanto en los servicios como en las manufacturas, situación que ha dado lugar al desplazamiento de trabajadores y la aparición de, lo que se ha dado en llamar, trabajo de consumo apenas abordado como tema de estudio (Glucksman, 2012).

e) La presión psíquica y el estrés laboral vienen a sustituir el deterioro físico como forma de desgaste principal de la fuerza de trabajo, característico de una gran parte de los procesos productivos del capitalismo industrial.

f) Pero el punto de quiebre más importante que da fisonomía al mundo del trabajo actual tuvo lugar a lo largo de la década de los noventa y, de alguna manera, imbrica los cambios señalados anteriormente. Se trata de la desaparición paulatina del empleo formal protegido, aquel caracterizado por la existencia de una relación clara entre el trabajador y su empleador, que generalmente contaba con protección social y prestaciones básicas; que 
trabajaba jornada completa en un establecimiento por lo que obtenía un salario "relativamente fijo"75.

En su lugar surgen y/o se expanden las relaciones salariales encubiertas, desarrollados bajo contratos temporales, de medio tiempo, por horas, de forma ocasional, carentes de protección social y prestaciones, con formas de pago variables e inciertas, en muchas ocasiones sin establecimiento o que usan como espacio de trabajo el hogar del mismo trabajador. Los procesos de externalización productiva y de subcontratación de mano de obra constituyen dos de las modalidades más utilizadas por el capital cuya lógica actual no requiere del empleo estable. En suma, el mundo del trabajo de hoy se caracteriza por el fin de la estabilidad laboral, la flexibilización y la extensión de la precarización del trabajo.

En A.L. las ocupaciones frecuentemente se habían ubicado en la esfera de la desregulación laboral. Esto es, los trabajos asalariados, formales, con prestaciones y seguridad social han constituido más la excepción que la regla. En la región proliferó, según el país y el momento, el trabajo realizado en pequeños establecimientos, con la participación de mano de obra familiar, incluso de niños y adolescentes ${ }^{76}$, sin protección laboral, o bien, el trabajo por cuenta propia y el crecimiento desmedido de las ocupaciones informales.

Informalidad que hoy se erige como uno de los problemas más apremiantes en esta región del mundo. De acuerdo a cifras recientes de la OIT (2011:67), ésta ha alcanzado niveles históricos a finales de la primera década del dos mil, pues 50 de cada 100 personas ocupadas tienen un empleo informal que, a su vez, se distribuyen de la siguiente manera: 33 de cada 100 ocupados se encuentran en el sector informal de la economía; 12 de cada 100 tienen un empleo informal dentro del sector de las empresas formales y alrededor de 5 de cada 100 se encuentran en el servicio doméstico.

Igual que la migración ocupacional, la informalidad ha sido considerada como una de las válvulas de escape al desempleo ante la ausencia de oportunidades laborales. Con la crisis de 2008-2009, se observa su crecimiento ${ }^{77}$ en la mayoría de los países de la región ${ }^{78}$. Para México, algunos autores (Samaniego, 2010) consignan su pérdida de funcionalidad por la saturación alcanzada, obligando a los buscadores de ocupación a volverse hacia otras opciones, como el regreso al trabajo agrícola o su incorporación a actividades vinculadas a la delincuencia y a la criminalidad, con el consabido peligro que estas representan, principalmente para los jóvenes.

Visto de otra manera, mientras que en Europa y Estados Unidos el trabajo se ubicó mayoritariamente en sectores de alta productividad, en su mayoría, protegidos; en América Latina, el trabajo se encontró en los de baja productividad (Weller, 2011), distinguiéndose por su alta precariedad a causa de la ausencia de protección social, bajos salarios, largas e intensivas jornadas, entre las características más sobresalientes.

Cabe señalar que en América Latina, ante la desprotección social en la que se encuentran sus habitantes por carencia de sistemas universales de protección, el peso de las redes sociales, encarnada, de forma destacada, más no exclusiva, en las familias, tradicionalmente ha jugado un papel preponderante, tanto como circuito de información

75En América latina, este segmento de empleo formal no tuvo el peso mayoritario como en Europa y Estados Unidos de Norteamérica, más que de forma muy localizada; se encontraba principalmente en el sector público, en las empresas paraestatales y en algunas grandes empresas privadas, algunas de ellas trasnacionales.

76En muchas regiones de América Latina, el trabajo infantil cumple otros cometidos de índole cultural pues constituye un medio de socializar conocimientos e iniciar en el trabajo a los indivíduos. Como ejemplo, véase a González de la Rocha y Escobar, 2008 para el caso de México, documentado a partir de la evaluación cualitativa realizada a su política social de los últimos años.

77Cabe señalar que a partir de la XVII Conferencia Internacional de Estadísticos del Trabajo, organizada por la OIT, en 2002, se amplía el concepto de sector informal, poniéndose acento en el puesto de trabajo, utilizando como criterio fundamental el acceso a la protección social sin importar la condición de asalariado o no.

78Ver en el anexo: cuadro “Composición de la Informalidad en países seleccionados de América Latina. 
como de apoyo en la búsqueda de oportunidades ocupacionales. Para el caso de Brasil, véase a Araujo Guimaraes, 2012.

Igualmente tales redes sociales constituyen un espacio de sustento para los trabajadores pobres, en los diversos eventos a lo largo de su vida, como los accidentes, enfermedades, incapacidades, embarazo, cuidado infantil, desempleo, muerte, etc. Cabe señalar que una gran parte de ellos se encuentran insertos en trabajos informales, situación que los vulnera como trabajadores y ciudadanos al grado de tener que enfrentar luchas diarias para ganarse el derecho al trabajo (Torres, 2011).

En este sentido, la situación de los trabajadores latinoamericanos se había diferenciado de los estadounidenses y europeos, donde el Estado y la institucionalidad laboral tradicionalmente habían tomado tal responsabilidad. La instauración del llamado Estado del Bienestar en esas regiones, después de la segunda guerra mundial, propició y sustentó el desarrollo del capitalismo industrial, cuyas generaciones de asalariados gozaron de estabilidad y protección laboral, posibilitándoles rechazar empleos insatisfactorios, gracias al apoyo decidido de las políticas públicas para desempleados en algunos países europeos (Auer, 2002).

Sin embargo, con la implementación de las políticas neoliberales en estos países, tales condiciones se han ido degradando tanto por el desmantelamiento del Estado de Bienestar como por la desregulación laboral, acercándose, de esta manera, a las condiciones laborales de los trabajadores latinoamericanos al compartir la precarización del trabajo como señal inequívoca de los tiempos que corren.

De cualquier forma, pese a las perspectivas del fin del trabajo (Rifkin, 2004), de la extinción de la clase obrera (Gorz, 1982) y del anunciado desplazamiento del trabajo por el consumo como centro de la cuestión social (Bauman, 2000), este sigue siendo referente sustancial en la vida de los individuos, constituye uno de los puntos neurálgicos de la sociedad global donde latinoamericana no es la excepción; actualmente enfrenta el ataque depredador del gran capital, el abandono de la regulación estatal, el retiro sindical y la regresión en los derechos laborales; el sometimiento abusivo a la tecnología y a las nuevas formas de organizar el trabajo y la producción, entre lo más importante.

\section{II - Tendencias actuales del mundo del trabajo en América Latina ${ }^{79}$}

Igual que para una gran parte del mundo, la escasez y precariedad de la ocupación son los signos del trabajo actual en América latina. La persistencia del desempleo y subempleo evidencia la mitad de esta afirmación, mientras la otra se expresa, por lo contrario, en extensas e intensivas jornadas de trabajo que sufre una gran parte de la población trabajadora, a cambio de salarios irrisorios y desprotección laboral ${ }^{80}$, como se verá más adelante.

De acuerdo a cifras de la OIT, durante el período 2008- 2011, el desempleo en los países seleccionados de la región latinoamericana alcanzó los niveles más altos en el año 2009 como una de las expresiones de la crisis económica desatada en los EEUU y la desaceleración

\footnotetext{
79Antes de iniciar este apartado conviene hacer dos señalamientos. El primero se refiere al motivo para hablar del "mundo del trabajo y no de mercados de trabajo". Obedece a que en estricto el último, es considerado como espacio de transacción del factor trabajo derivado de relaciones salariales, mientras que el mundo del trabajo engloba también a los individuos que desempeñan una actividad por su cuenta que les procura ingresos, en ocasiones no monetarios. En este sentido y dado el protagonismo de la última modalidad de ocupación en América Latina, hablaré de mundo del trabajo aunque hay que reconocer que no cuenta con la fuerza conceptual que el de mercado de trabajo. El segundo señalamiento se refiere al universo de análisis en esta sección, se trata de seis países de la región latinoamericana: Argentina, Brasil, Chile, Colombia, México y Perú. Pese a su importancia Venezuela, Bolivia y Ecuador no son abordados en este texto por falta de información estadística medianamente confiable. Se trata del grupo "revolucionario" de A.L. caracterizado por la implementación del Estado "compensador" de acuerdo a autores como Stefanoni, 2012.

80La precarización del trabajo es un concepto multidimensional referido a las condiciones endebles de contratación; al uso intensivo y flexible de la mano de obra; a los riesgos psicosociales derivados de la organización del trabajo; a las deficientes condiciones de trabajo; a la implementación de mecanismos de retribución variable; a la ausencia de protección social, etc. que puede resumirse en inestabilidad e incertidumbre laboral para quienes lo padecen.
} 
subsiguiente de los demás países europeos y asiáticos como China quienes disminuyeron sus importaciones de productos latinoamericanos. Destaca Colombia como el país con mayor desempleo (dos dígitos), seguido de Argentina, Brasil, Chile, Perú y México; resalta también que aunque todos muestran una disminución del desempleo en 2011, México y Colombia no pudieron retornar a las cifras de 2008. Visto por sexos, a excepción de México, son las mujeres quienes registran las cifras más altas de desempleo en relación a los hombres.

Asimismo, es la población joven (de 15 a 24 años) el segmento etario más afectado por la falta de ocupación, alcanzando dos dígitos en 2011 en todos los países. Conviene señalar que Argentina, Brasil, Chile y Colombia registraron disminuciones en este rubro, de 2010 a 2011, mientras que México y Perú, por el contrario, aumentaron sus tasas de desempleo juvenil ${ }^{81}$.

En cuanto a la protección de los trabajadores en los rubros de salud y/o pensiones, en los años 2008 y $2010^{82}$, es Colombia quien se encuentra a la cabeza con más del $80 \%$ de su población ocupada con acceso a tales protecciones, mostrando un ligero incremento de un año a otro; le sigue Argentina con 70.5\% y 72.6\% de ocupados con protección en los dos años revisados respectivamente, evidenciándose, como en el caso de Colombia, un incremento de 2008 a 2010. Con menor cobertura (63.8\%) se encuentra Brasil en 2008; mientras Perú, en el mismo año, solamente registra a la mitad de sus trabajadores con acceso a tales protecciones. Sin embargo, en 2010 su incremento es notable al alcanzar al 62.4\% de ellos. De todos los países analizados, México es el que registra menor cobertura de protección en tales rubros pues la protección en salud y pensiones para sus trabajadores no llega ni a la mitad, apenas cubre al $48.9 \%$ en 2008 y al $46.2 \%$ en 2010 , aunque para este último año la cifra únicamente incluye a salud y no a las pensiones al no contarse con esta información.

Sin embargo, cuando se analiza con mayor detalle los componentes de estas cifras, inmediatamente destaca la polarización de las condiciones de empleo entre países y segmentos de trabajadores, corroborándose algunas de las tendencias señaladas en el apartado anterior. Así, aunque con pequeñas diferencias entre países, es en el sector público donde se concentran los trabajadores con acceso a salud y pensiones. No así en el sector privado y menos cuando se trata de establecimientos de 5 o menor número de trabajadores, cuya cobertura, a excepción de Colombia ( $80 \%$ ) y, en menor medida, de Argentina $(57 \%$ en 2010), es de menos de la mitad de los trabajadores ahí ubicados. Destaca el caso de México con apenas el $17.8 \%$ y $16.1 \%$, en 2008 y 2010 respectivamente, de trabajadores protegidos en el sector privado, en establecimientos con 5 o menos trabajadores.

En el caso de los ocupados no asalariados, vistos en conjunto, la tasa de protección desciende considerablemente respecto a los asalariados, confirmándose que en América Latina, ante la ausencia de sistemas universales de protección, el trabajo asalariado constituye la vía principal de acceso a ella, lo que implica la desprotección de la otra parte de los ocupados en otra condición. Entre países también se observan las diferencias; Colombia se mantiene con mayor porcentaje -cercano al 90\%- de protección de sus no asalariados e incluso presenta un crecimiento de 2008 a 2010; le sigue Argentina con alrededor del 60\%, aunque se observa una leve disminución en 2010. En el caso de Brasil, los trabajadores en la misma situación apenas rondan el 40\% mientras que en Perú se incrementan del $46 \%$ en 2008 al 63\% en 2010. Nuevamente México queda al final al registrar prácticamente a todos sus trabajadores no asalariados sin protección, más grave en el caso de las pensiones, apenas un $2.1 \%$ de este grupo de ocupados contaban con tal protección en 2008.

81 Cabe señalar que en la región latinoamericana, México es el país con peor desempeño ante la crisis de 2008. De acuerdo al Boletín de la CEPAL/OIT, México es el país que en 2009 mostró el impacto más inmediato y notorio de la crisis en el mercado laboral sin visos de una pronta recuperación.

82 Según cifras de la OIT 
Cuando se analiza la composición de los no asalariados ${ }^{83}$, destaca el segmento de los patrones donde un alto porcentaje declaró contar con protección; nuevamente Colombia, Argentina y Perú son los mejor posicionados en este rubro, en Perú, alrededor de la mitad de los patrones están protegidos. México se ubica al final del listado pues ni los patrones cuentan con protección, apenas el $1.3 \%$ registraron con protección en salud y pensiones en 2008 y el .3\% únicamente en salud en 2010.

Los otros dos grupos que conforman el segmento de no asalariados son los trabajadores independientes, que incluye a los familiares auxiliares, y los trabajadores del servicio doméstico. En ambos grupos, la cobertura de protección en los países analizados sigue la misma tendencia: Colombia a la cabeza con alrededor del $80 \%$ de trabajadores con acceso a salud y pensiones en el primer grupo y por encima del $80 \%$ en los trabajadores domésticos. Inmediatamente se ubica Argentina, seguida de Perú. En Argentina, la mitad de sus trabajadores independientes se registraron con protección en los rubros señalados, aunque se observa una leve caída de 2008 a 2010, mientras que, por el contrario, en el servicio doméstico se amplía la población con protección al pasar de 43.4\% en 2008 a 54\% en 2010. Por su parte, Perú muestra una mejoría en protección en los dos grupos analizados aunque la cobertura es significativamente menor que la registrada en los países precedentes: $38.6 \%$ para los independientes en 2008 y $53.7 \%$ en 2010 ; mientras que para los trabajadores domésticos, la protección se ubicó en $29.1 \%$ en 2008 y 45.4\% en 2010. La protección para los trabajadores independientes mexicanos es prácticamente inexistente, apenas de un $1.9 \%$ en 2008 y . $1 \%$ en 2010 , mientras que en los trabajadores del servicio doméstico la protección alcanzó al 6.9\% en 2008, disminuyendo a 6.4\% en 2010.

En cuanto a la informalidad, calculada con respecto al total del empleo no agrícola, destaca sus altos porcentajes por encima de la mitad de la población trabajadora, a excepción de Argentina que en 2011 disminuyó al 46.7\%.

Las cifras más altas de informalidad, por encima del 70\%, son las de Perú; le sigue Colombia con un ligero incremento del 2008 (58.4\%) al 2010 (59.6\%); igualmente México presenta un comportamiento ascendente al pasar de $52.5 \%$ en 2008 a $54.2 \%$ en 2010. De acuerdo a la conceptualización actual de la OIT, la informalidad se integra tanto por trabajadores del sector informal como del formal, así como con los trabajadores del servicio doméstico. El criterio definitorio para ser considerado como tal es la ausencia de protección social.

Respecto a este último grupo de trabajadores, cabe hacer notar, que la aprobación en 2011 del Convenio 189 de la OIT acerca del trabajo decente para las y los trabajadores domésticos constituye un primer paso en la larga lucha por el respeto y reconocimiento de tales trabajadores, con ello se espera pueda mejorar la situación laboral de este grupo ocupacional, ubicado en los hogares y tradicionalmente mal pagados, sin horario establecido, desvalorados y desprotegidos. La mejoría alcanzaría no solamente a los trabajadores domésticos de América Latina, sino también a los migrantes latinoamericanos que se desempeñan como trabajadores del hogar en otros países, pues dicho convenio proporciona las normas mínimas de protección al trabajo doméstico ${ }^{84}$.

Para todos los países revisados, el grueso de los trabajadores informales se encontraron en el mismo sector informal aunque las cifras de los ubicados en el sector formal

83Los no asalariados están conformados por los patrones, los trabajadores independientes y familiares auxiliares, y los trabajadores del servicio doméstico.

84Entre los propósitos de la Conferencia 100 referido al trabajo doméstico se encuentra: el reconocimiento al trabajo doméstico como contribuyente a la economía nacional y mundial; reconocimiento como trabajo formal y sus trabajadores tienen derechos iguales a los demás trabajadores; establecimiento de un horario de trabajo; regulación de las agencias temporales de empleo; un marco que permita garantizar la seguridad social, así como la salud y seguridad en el trabajo y un marco para la protección de los trabajadores migrantes. 
va de $18.8 \%$ para Perú en 2008, 15.3\% para México, 13.1\% en Argentina y $6.4 \%$ en Colombia presentando una ligera disminución en todos los países, en 2010.

Una revisión de este tipo no estaría completa sin la exploración del papel de los sindicatos y los ingresos. Sin embargo, las estadísticas existentes presentan problemas que dificultan su comparación y su confiabilidad. Para el caso de los sindicatos, se puede decir que en general fueron impactados negativamente por la reducción de la demanda de fuerza de trabajo y del empleo formal, especialmente en el sector público por las privatizaciones de empresas estatales que tuvieron lugar en los años noventa y dos mil; también fueron afectados tanto por la descentralización y externalización del trabajo como por el repunte del trabajo a domicilio. En conjunto, tales hechos impactaron principalmente el nivel de sindicalización, el poder de interlocución y la legitimidad, esta última derivada en gran medida, por el carácter clientelar que ha caracterizado a una parte del sindicalismo de la región.

Pese al panorama adverso, en algunos países se ha registrado un ligero repunte en las tasas de sindicalización durante la década de los dos mil, situación documentada en diversos estudios, principalmente para los casos de Argentina (Sénen, 2011) y Brasil (Dari K., José, 2012). No así en Venezuela donde la tasa de sindicalización disminuyó del 2000 al 2010 a pesar del crecimiento en el número de sindicatos (Iranzo, 2011) ni en Chile donde la transición de la dictadura militar a la democracia muestra un sindicalismo debilitado (Aravena y Núñez, 2011). En el caso de México, el desprestigio de los sindicatos corporativos y, en general, el escaso poder sindical queda de manifiesto en la reciente arremetida neoliberal, contra derechos adquiridos por los trabajadores, mediante la reforma laboral finalmente aprobada, después de varios intentos fallidos, a finales de 2012.

En cuanto a los ingresos de las poblaciones latinoamericanas, éstos se caracterizan por su enorme desigualdad, en términos de su concentración en pocas manos en detrimento de una gran mayoría con ingresos, provenientes del trabajo, extremadamente bajos. De acuerdo a la OIT (citado por Jûrgen, 2012:138) la décima parte más rica ya concentra hasta el 50\% de los ingresos nacionales. El mismo autor aclara: "la inequidad extrema no solo se manifiesta en términos de ingreso y patrimonio, sino que también se refleja en un dispar acceso a la tierra y a bienes públicos esenciales como la educación, la salud o la seguridad social."

En este contexto, desde la década de 2000 cobran relevancia la multiplicación de programas sociales de transferencias públicas condicionadas, de tipo asistencial, orientadas a la población pobre, (Barrientos, 2012). Se trata de programas implementados en gran escala de transferencia directa de ingresos, dirigidos a hogares en situación de pobreza y extrema pobreza. Junto con ello, las cuantiosas remesas de los migrantes es lo que explica la sobrevivencia de una amplia capa de la población latinoamericana.

\section{III - Reflexión final}

Si bien el trabajo sigue habitando en el centro de la vida individual y social, el deterioro, sometimiento y degradación en el que se encuentra actualmente es innegable como también lo son las numerosas voces que se han alzado, por diferentes rincones del mundo, para denunciar tal situación y demandar puestos de trabajo de calidad. A su vez, los efectos en las personas pueden encontrarse en las crónicas hemerográficas del día a día o simplemente mirando a nuestro alrededor.

En el marco de la globalización, se constata una regresión de los derechos laborales conquistados que amenaza el futuro de las nuevas generaciones de trabajadores arrojándolos a la incertidumbre; que si bien la escasez de ocupación constituye uno de los signos actuales 
del mundo del trabajo, la intensificación que enfrentan los ocupados caracteriza el trabajo de hoy, tanto en la producción material como en la inmaterial. Largas jornadas, realización de varias tareas a la vez, demanda de mayor rapidez, vigilancia extrema, supresión de descansos, entre otras cosas, se han convertido en los componentes principales que acompañan al trabajo en su realización.

En el ámbito de la retribución, es el salario por piezas, comisiones, en especie, vinculados a diversos factores (productividad, asistencia y puntualidad, y otros) lo que conforma la variabilidad e incertidumbre en los ingresos de la clase trabajadora. Asimismo, los inesperados cambios de horario, lugar de trabajo, recortes de personal, supresión de derechos laborales, contribuyen a la precarización del empleo.

Todo ello se traduce en una notoria disminución en la demanda de trabajo humano para la producción de bienes y servicios. De esta manera, la inestabilidad hace presa a los hombres y mujeres que viven de su trabajo, tanto en lo individual como en lo colectivo, alimentando así la vulnerabilidad, pobreza y desigualdad en la región latinoamericana aunque de forma heterogénea y diferencial en relación, entre otras cuestiones, a su historia socioeconómica, al desempeño de los sindicatos, de sus Estados y la naturaleza y alcance de su política social.

En América Latina, sus habitantes enfrentan tal panorama desolador mediante la informalidad y la migración internacional pese a que ambas están llegando a su límite como opciones viables; la primera por la saturación que presenta, mientras que la segunda, por situaciones externas como la contracción de la economía norteamericana con la consabida expulsión de mano de obra.

Por todo lo señalado, el trabajo constituye el centro neurálgico de la dinámica social contemporánea susceptible de agudizar los conflictos sociales. De aquí la necesaria participación de todos, cada uno desde su espacio, para evidenciar la falsa inevitabilidad de la degradación laboral en el marco de la llamada globalización y exigir transformaciones de fondo para el beneficio de todos, principalmente de los más desprotegidos.

\section{Submetido em 3 de dezembro de 2012 e aceito para publicação em 10 de setembro de 2013.}

\section{Referências}

ANTUNES, Ricardo (2012) La nueva morfología del trabajo y sus principales tendencias. Informalidad, infoproletariado, (in) materialidad y valor. En Sociología del trabajo, no. 74, nueva época, invierno. Siglo XXI, España editores. España.

ARAUJO GUIMAREAS, Nadya (2012) ¿Cómo salir del desempleo? Lazos fuertes y lazos débiles en la búsqueda de trabajo en Sao Paulo, Brasil. En Sociología del Trabajo, nueva época, no. 74, invierno. Siglo XXI España editores, España.

ARAVENA, Antonio y Núñez, Daniel (2011) Los gobiernos de la Concertación y el sindicalismo en Chile en Revista Trabajo, año 5, no. 8, julio-diciembre, tercera época. UAM Iztapalapa, OIT, Plaza y Valdés editores. México

AUER, Peter (2002) La recuperación del empleo en Europa. El ejemplo de Austria, Dinamarca, Irlanda y los países bajos. OIT, Ginebra.

BAUMAN, Zygmunt (2000) Trabajo, consumismo y nuevos pobres. Gedisa, Barcelona, España.

BARRIENTOS, Armando (2012) Dilemas de las políticas sociales latinoamericanas chacia una protección social fragmentada? Nueva Sociedad, no. 239, mayo-junio. Fundación Friedrich Ebert, Buenos Aires, Argentina. 
BOUTILLER, Sophie y Beatriz Castilla (2012) La precarización del mercado de trabajo: análisis desde Europa, América Latina y el Caribe. Papeles de Población. Vol. 18, no. 71, enero-marzo. Universidad Autónoma del Estado de México, Toluca, México.

CASTEL, Robert (1995) La metamorfosis de la cuestión social. Una crónica del salariado. Paidós. Argentina.

(2010) El ascenso de las incertidumbres. Trabajo, protecciones, estatuto del individuo. FCE. Argentina.

CEPAL/OIT (2009) Boletín Coyuntura laboral en América Latina y el Caribe, no. 2, Crisis en los mercados laborales y respuestas contracíclicas. Septiembre.

CEPAL (2010) Panorama social de América Latina 2010, CEPAL, Santiago de Chile

COHEN, Daniel (2001) Nuestros tiempos modernos. "Un análisis del capitalismo y sus tendencias: ¿estamos ante el final del trabajo? Kriterios TusQuets editores, España.

CORAGGIO, José Luis (1999) Política social y economía del trabajo. Alternativas a la política neoliberal para la ciudad. Universidad Nacional de General Sarmiento. Miño y Dávila editores. España.

CORTÉS, Fernando; Escobar, Agustín y De la Rocha, Mercedes (2008) Método científico y política social. A propósito de las evaluaciones cualitativas de programas sociales. COLMEX, México, D.F.

CORIAT, Benjamìn (1979) El taller y el cronómetro. Ensayo sobre el taylorismo, el fordismo y la producción en masa. $2^{\mathrm{a}}$ edición. Siglo XXI, México.

CORIAT, Benjamín (1992) Pensar al revés. Trabajo y organización en la empresa japonesa. Siglo XXI editores, Madrid, España.

DARI KREIN, José y Sos Santos, Anselmo L. (2012) La formalización del trabajo en Brasil. El crecimiento económico y los efectos de las políticas laborales. Nueva Sociedad no. 239, mayo-junio. Fundación Friedrich Ebert, Buenos Aires, Argentina.

DE SOUSA SANTOS, Boaventura (2010) Refundación del estado en América latina. Perspectivas desde una epistemología del Sur. Universidad de los Andes, Siglo XXI editores, Buenos Aires. Argentina.

GONZÁLEZ DE LA ROCHA, Mercedes y Escobar Latapí, Agustín (2008) Vulnerabilidad y activos de los hogares: El Programa Progresa-Oportunidades en ciudades pequeñas en Córtes Fernando, Escobar, Agustín y González, Mercedes. Método científico y política social. A propósito de las evaluaciones cualitativas de programas sociales. El Colegio de México. México.

GLUCKSMAN, Miriam A. (2012) Formaciones socioeconómicas de trabajo y el trabajo del consumo en Sociología del Trabajo no. 75, primavera. Siglo XXI España editores, Madrid, España.

HARVEY, David y otros (2012) Occupy. Boitempo Editorial, Carta Maior. Sao Paulo, Brasil.

IRANZO, Consuelo (2011) Chávez y la política laboral en Venezuela 1999-2010 en Revista Trabajo, año 5, no. 8, julio-diciembre, tercera época. UAM Iztapalapa, OIT, Plaza y Valdés editores. México.

MIDAGLIA, Carmen (2012) Un balance crítico de los programas sociales en América Latina. Nueva Sociedad no. 239, mayo-junio. Fundación Friedrich Ebert, Buenos Aires, Argentina.

OIT (2006) El Consenso de Mar del Plata. IV Cumbre de las Américas 2005. Análisis y Perspectivas. Oficina Regional para América Latina y el Caribe, Buenos Aires, Argentina.

---- (2011) Panorama laboral 2011. Oficina Regional para América Latina y el Caribe. Perú.

---- (2012) Panorama laboral 2012. Oficina Regional para América Latina y el Caribe. Perú.

QUIJANO, Aníbal (s/f) Colonialidad del poder, eurocentrismo y América Latina.

RIFKIN, Jeremy (2004) El fin del trabajo. Nuevas tecnologías contra puestos de trabajo: el nacimiento de una nueva era. $9^{\circ}$ edición. Paidós, España.

SAMANIEGO, Norma (2010) El empleo y la crisis. Precarización y nuevas “válvulas de escape” en Economía UNAM no. 20, mayo-agosto. UNAM, México, D.F.

SÉNEN, Cecilia (2011) La revitalización sindical en Argentina durante los Kirchner. Revista Trabajo, año 5, no. 8, julio-diciembre, tercera época. UAM Iztapalapa, OIT, Plaza y Valdés editores. México. 
STEFANONI, Pablo (2012) Posneoliberalismo cuesta arriba. Los modelos de Venezuela, Bolivia y Ecuador a debate en Nueva Sociedad 239, mayo-junio, Fundación Friedrich Ebert, Buenos Aires, Argentina.

TORRES, Beatriz (2011) "El trabajo actual: entre la escasez y la precariedad" en Beatriz Castilla y Beatriz Torres (editoras) Tras las huellas del trabajo: de la firma red a los "otros trabajos". Universidad Autónoma de Yucatán. Mérida, Yucatán, México.

(2011) (coordinadora) Experiencias y estrategias de protección social desde organizaciones de trabajadores informales urbanos en México: los casos de las ciudades de México, Monterrey y Mérida. Informe de investigación. WIEGO, IIE, UNAM, CIS, Mérida, Yucatán, México. WELLER, Jurgen (2011) Panorama de las condiciones de trabajo en América Latina en Nueva Sociedad, no. 232, marzo-abril. Fundación Friedrich Ebert, Buenos Aires, Argentina. 